\title{
Analog Circuits for Thermistor Linearization with Chebyshev-Optimal Linearity Error
}

\author{
Carl Renneberg \\ Tau Research \\ Sydney, NSW, Australia \\ carl.renneberg@science-logic.net
}

\author{
Torsten Lehmann \\ School of Electrical Engineering and Telecommunications \\ University of NSW \\ Sydney, NSW, Australia \\ tlehmann@unsw.edu.au
}

\begin{abstract}
This paper investigates analog linearization circuits for NTC thermistors, and the theoretical limits to linearity performance. In theory, by using multiple identical thermistors, the circuit may have a linearity error that is arbitrarily small and optimal in the Chebyshev norm. This result also applies to many other impedance-type sensors (e.g. resistive, capacitive). Experimental data from a three-thermistor circuit shows approximately $\pm 16 \mathrm{mK}(160 \mathrm{ppm})$ linearity over a span of 100 ${ }^{\circ} \mathrm{C}$, consistent with a theoretical limit of $\pm 12 \mathrm{mK}(120 \mathrm{ppm})$.
\end{abstract}

\section{INTRODUCTION}

NTC thermistors are widely used in industry, for temperature measurement and in temperature compensation circuits. These devices are sensitive but non-linear (e.g. fig. 1). Although digital methods are wide-spread, analog methods for thermistor linearization and temperature compensation are useful in cost-sensitive applications. One analog approach is to use multiple thermistors so that the overall circuit synthesizes a desired output-versus-temperature function with acceptable accuracy. Often the desired function is linear, as in electronic thermometers [1][2], or constant, as in TXCOs [3][4]. This concept, of combining multiple similar non-linear devices, is used in other areas of circuit design, such as linearization of amplifiers and mixers [5], and varactors [6]. It is natural to ask, what are the theoretical limits to linearity performance in analog linearization circuits? Is it possible to get close to those limits in practical, cost-effective designs? In the field of electrical filters, questions about performance limits and their realization are answered by an extensive literature; in the field of linearization and compensation circuits, they are not.

In this paper, we investigate the first question, as it applies to NTC thermistors. We show that, in principle, the linearity error of multiple thermistor circuits can be made arbitrarily small and optimal in the Chebyshev (uniform) norm. This result also applies to many other impedance-type sensors (e.g. resistive, capacitive) and is believed to be new. The theory has important implications for practical design; we propose and discuss some design rules. Despite self-heating effects, measurement data taken from a three-thermistor circuit show about $\pm 16 \mathrm{mK}$ (160 ppm) linearity over a span of $100{ }^{\circ} \mathrm{C}$, consistent with an ideal limit of $\pm 12 \mathrm{mK}(120 \mathrm{ppm})$.
Simulation results suggest that, as the number $n$ of thermistors increases, the optimal linearity error decreases exponentially.

\section{Best Approximation In the Sensor Domain}

\section{A. Outline of the Theory}

Fig. 1 shows the nominal characteristics of thermistor type YSI 45008 [7]. Fig. 2 shows a circuit with $n \geq 1$ thermistors, all at the same temperature. Output signal $V_{\text {out }}$ equals a weighted sum of thermistor voltages. Assume self-heating effects are negligible, and the $n$ thermistors have identical characteristics. Then the normalized output $S=V_{\text {out }} / V_{\text {ref }}$ can be written as a rational function of thermistor resistance $x$ :

$$
S(x)=\sum k_{i} x /\left(R b_{i}+x\right)
$$

Ideally, $S$ closely approximates a desired continuous function $f$ of thermistor temperature $T$, to within a constant scale factor $c$ and offset $d$ :

$$
S \approx c f(T)+d
$$

For linearization circuits, $f(T)=T$. Because the nominal resistance function in fig. 1 is strictly monotonic, $T=g(x)$, for some function $g$. Using the idealized relationship in (2), the value $f(T)_{e s t}$ estimated by the circuit output is given by:

$$
f(T)_{e s t}=(S(x)-d) / c
$$

The approximation error in $f(T)_{e s t}$ is given by:

$$
f(T)_{e s t}-f(T)=[(S(x)-d) / c]-f(g(x))
$$

On the right-hand side (r.h.s) of (4), the first term, in square brackets, is a rational function of thermistor resistance $x$; the second term, $f(g)$, is a continuous real function of $x$. The error in (4) is minimized if the first r.h.s. term is a best rational approximation to the second r.h.s. term, over the temperature range of interest. 
Some results from approximation theory apply directly to circuits such as fig. 2. In particular, if one seeks to minimize the linearity error in the uniform norm, then a best rational function exists, and is unique (when expressed in a canonical form). Furthermore, the approximation error of the best rational function has the following alternation property [8][9].

Let $\partial Q$ denote the degree of polynomial $Q$. Let $\|Q\|$ denote the norm of $\mathrm{Q}$. Consider the class $C$ of rational functions $P(x) / Q(x)$ bounded on interval $[a, b]$, where $\partial P \leq n$, $\partial Q \leq m$, and $P / Q$ is irreducible (no roots in common). Function $P(x) / Q(x)$ has degree $(\partial P, \partial Q)$. Without loss of generality, normalize $P / Q$ so that $Q(x)>0$ and $\|Q(x)\|=1$ (for example). Let function $g$ be continuous on $[a, b]$. In order for $P / Q$ to be the best approximation from class $C$ to $g$ in the uniform norm, over $[a, b]$, it is necessary and sufficient that the error function $E=g-P / Q$ have at least $N$ alternating points $x_{0}$, $x_{1} \ldots x_{N-1}$ as follows:

$$
\begin{gathered}
N=2+\max [n+\partial Q, m+\partial P] \\
E\left(x_{i}\right)=-E\left(x_{i+1}\right)= \pm\|E\|, a \leq x_{0}<x_{1}<\ldots x_{N-1} \leq b
\end{gathered}
$$

The Weierstrass theorem implies $\|\mathrm{E}\| \rightarrow 0$ as $\partial P \rightarrow \infty$ [18].

\section{B. Practical Design Principles}

Several practical design principles follow from (5) and (6).

1) $2 n+2$ Alternations: Typically, $N=2 n+2$ for an $n$ thermistor circuit. The reasons are two-fold. Firstly, the use of $n$ sensors generally results in a rational function of degree ( $n$, $n$ ) in (4) (the r.h.s. term in square brackets). Secondly, the best $(n, m)$ approximation to $f(g)$ in the uniform norm often has $N=$ $n+m+2$. Unless there is reason to believe $f(g)$ has a lower value of $N$, the designer should initially search for a solution that has at least $2 n+2$ alternations in the error function. (See the discussion of "normal" and "hypernormal" functions in [9]).

2) $2 n+1$ Degrees of Freedom: Since it has $N$ alternations, the optimal error function will have $N-1$ distinct zeroes. The circuit must have at least $N-1$ (typically $2 n+1$ ) degrees of freedom, in component values, circuit parameters, etc., so that the circuit can locate these zeroes at the best values of thermistor resistance (or temperature). This rule is crucial to achieving near-optimal results in practice. Since the best uniform approximation is unique, any deviation from the optimal zero locations must increase the linearity error.

3) 2n-1 Circuit Parameters: While the circuit must have at least $\mathrm{N}-1$ degrees of freedom, two of those may reside in parameters $c$ and $d$ in (4). If these two values are allowed to vary, then an optimal circuit must have at least $N-3$ (typically $2 n-1)$ degrees of freedom in its components and other parameters. This principle is useful when the designer seeks to implement an optimal circuit with minimal component count.

\section{Examples}

The linearization examples in figures 3-6 illustrate the design principles above. Fig. 3 shows the calculated optimal error function for figures 1 and 2 , when $n=3$; as per the $2 n+2$ rule, the number of alternations equals $2 n+2$. Fig. 4 shows how the calculated optimal error varies with $n$. For this particular sensor (fig. 1) and temperature range, the error decreases by a factor about 18 for each increment in $n$.

Fig. 5(a) shows a two-thermistor linearization circuit from [1], used commercially [7][10]. The thermistors in Fig. 5(a) have different characteristics - see YSI 44018 dual-thermistor data sheet ([7]) - but given the $2 n-1$ rule, one might suspect that the circuit is not optimal. This happens to be the case. When R1 and R2 equal the recommended values for the YSI 44018 , for the temperature range -5 to $45^{\circ} \mathrm{C}$, the linearity of fig. 5(a) is quoted as $\pm 65 \mathrm{mK}$ ([7]). Fig. 5(b) shows a modified circuit that uses the same dual-thermistor device (but transposed); an extra resistor increases the degrees of freedom to the suggested minimum. Fig. 6 shows that the modified circuit has a calculated linearity error five times lower.

\section{Discussion}

Electrical circuits naturally give rise to rational transfer functions. According to (4), the circuit should implement a best rational approximation to $f(g)$, the desired transfer (temperature) function applied to the inverse sensor function. The best approximation in the uniform norm is unique, but it can be realized by a variety of circuit topologies [11] (reminiscent of filter synthesis).

The derivation of (4) assumes that the circuit uses the same sensor type; that the sensors are impedance-type (e.g. resistive, capacitive) and electrically linear; and that the sensor characteristic is strictly monotonic. So it applies to many sensor types, e.g. RTDs and spreading-resistance temperature sensors [12]. As per figures 5(b) and 6, discussed above, the assumption of multiple identical sensors can often be relaxed. Furthermore, one can transform a multi-sensor circuit such as fig. 2 into a single-sensor circuit that performs the weighted summing of signals sequentially (e.g. digitally) [11]. The single-sensor circuit has the same linearity as one with multiple sensors that are perfectly matched. Hence a circuit design that uses multiple identical sensors can serve as a basis for other, application-specific designs.

In figures 2 and 5(b), the optimal fixed resistor values generally will not equal preferred values (e.g. E96). Within certain limits, arbitrary resistor values can be implemented using a polysilicon-based trimming technology [13][14].

\section{E. Calculating the Component Values}

Circuit values can be derived in various ways. One method [11] we have found to be versatile, and have used throughout this paper, is: (a) calculate $x$, the sensors' resistance (or capacitance) as a function of temperature at many (e.g. 100) discrete points throughout the temperature range; (b) write a MATLAB [15] or similar software routine that evaluates the circuit's transfer function at the discrete points, given the component values as input; (c) use the Nelder-Mead simplex algorithm [17] to search for component values that minimize the transfer function's approximation error. While intensive computationally, this approach caters for constrained searches: one can easily include penalty functions in the objective function, to allow for any design constraints that have priority 
over strict optimality. It also caters for non-identical sensors, and for temperature effects in other circuit components. Algorithms that compute best rational approximations (e.g. see [8]), can be used in alternative methods. To model thermistor resistance $x$, one can first express $\log (x)$ as a polynomial in $1 / T$ [16], using measured values or manufacturer's data to calculate the polynomial's coefficients.

\section{EXPERIMENTAL RESULTS}

This section summarizes verification tests conducted on the three-thermistor linearization circuit of fig. 7. The circuit used Betatherm thermistors type 30K6A (30 Kohms nominal at $25^{\circ} \mathrm{C}$ ). The three devices were calibrated together in a Hart Scientific dry block calibrator, type 9107, over the range 0 $100{ }^{\circ} \mathrm{C}$. During calibration, the thermistor resistances were measured with an Agilent 6 $1 / 2$ digit DVM, type 34401A, via 4wire connections. The dry well temperature was measured near the thermistor packages, using a Hart Scientific data logger type 1522 ( $1 \mathrm{mK}$ resolution) and thermistor probe type 5611T. The thermal resistance of each device package at 58 ${ }^{\circ} \mathrm{C}$ was calculated using thermistor resistance data taken with two DVM measurement currents. Raw thermistor data were corrected for self-heating effects due to DVM current, then a nine-term polynomial (as above) was fitted to the data for each thermistor. The circuit values for optimal linearity of the terminal resistance were found using the Nelder-Mead algorithm (as above). The circuit was constructed, then the thermistors were connected into the circuit (located outside the dry well), without otherwise disturbing the test setup.

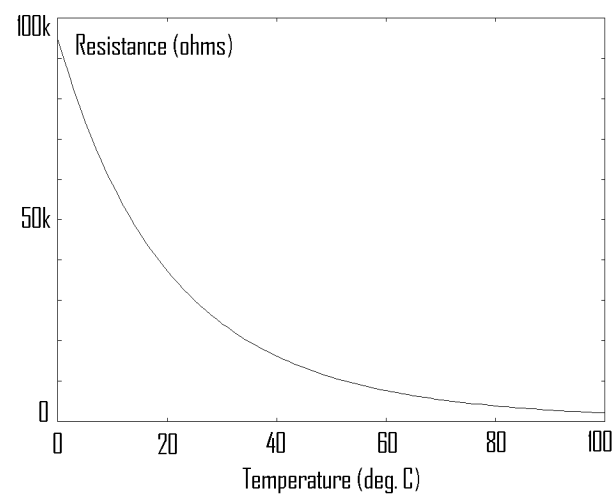

Figure 1. Nominal resistance versus temperature for YSI 45008.

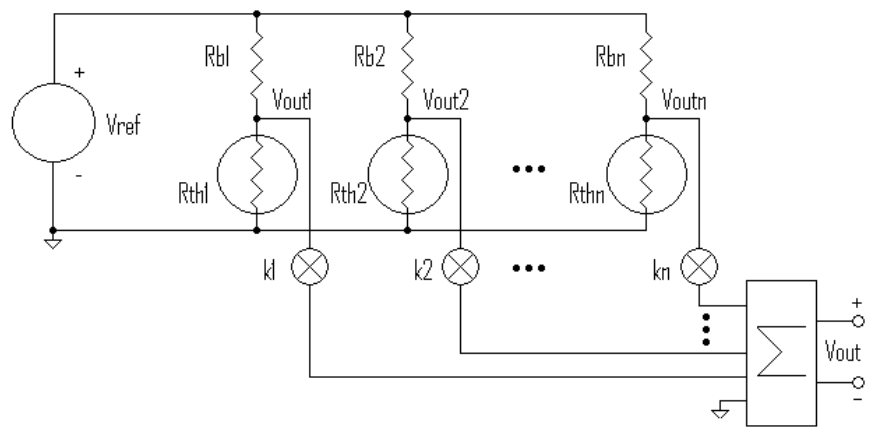

Figure 2. An $n$-thermistor circuit illustrating the rational approximation principle.

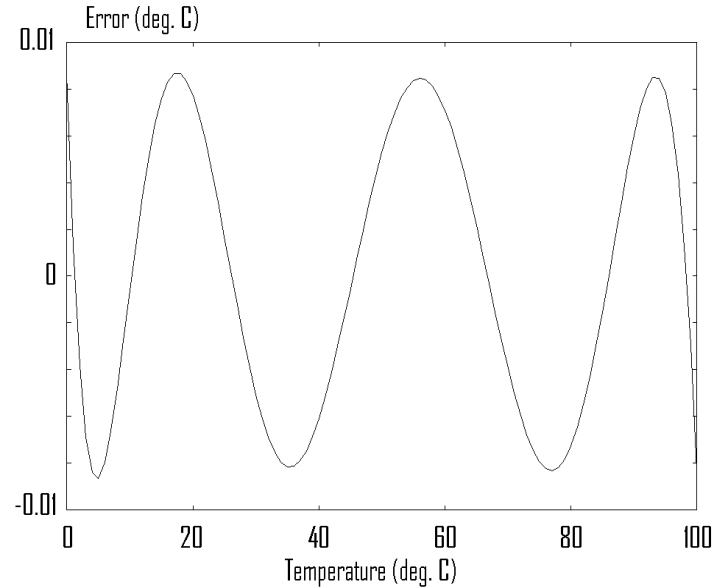

Figure 3. Calculated optimal error function for fig. 2, $n=3$.

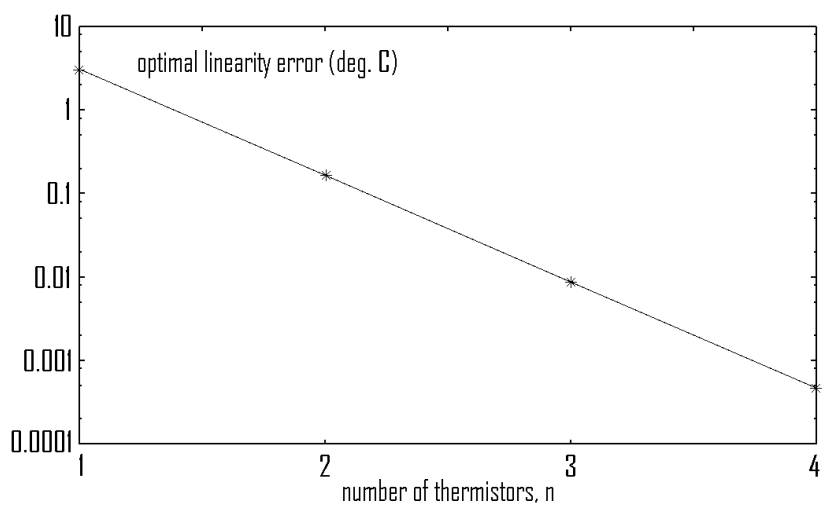

Figure 4. Calculated optimal linearity error versus $n$ for fig. 2 .

(See fig. 8 for the actual values used.) The circuit's resistance (4-wire connection) was then measured, using the same equipment and temperature settings used for the calibration run. Fig. 8 shows the raw terminal resistance versus temperature of the circuit under test. Fig. 9 shows the raw measured linearity error, with peaks of about $\pm 16 \mathrm{mK}$. The calculated optimal error for fig. 9 was $\pm 12 \mathrm{mK}$. Of the discrepancy between theoretical and measured linearity, \pm 2 $\mathrm{mK}$ is due to tolerances in the fixed resistances. Fig. 9 shows the characteristic pattern of $2 n+2$ alternations expected from a near-optimal three-thermistor circuit, in agreement with the theory. Note that figures 8 and 9 include self-heating effects.

\section{CONCLUSIONS}

This paper has proposed the application of rational approximation to the design of analog circuits for thermistor linearization. The main theoretical result is that the linearity error can be made arbitrarily small and optimal in the Chebyshev norm, by using multiple thermistors of the same type. This result also applies to a variety of other sensor types. Simulation results shown here suggest that the requirement for near-identical devices can be relaxed in practice, and that it may be possible to generalize the theory to non-identical sensors. Experimental results show good agreement with the theory, despite thermistor self-heating in the circuit under test. 

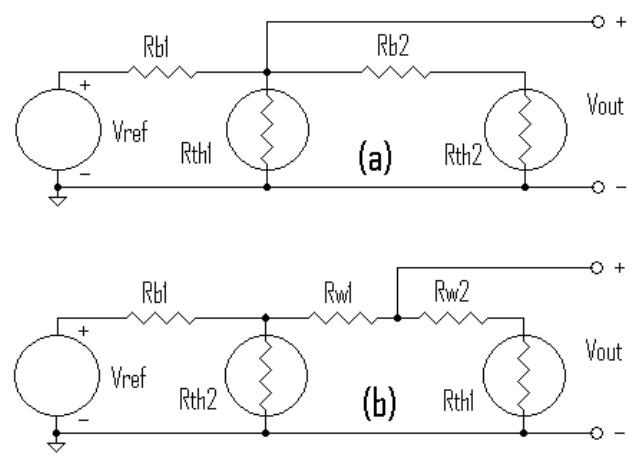

Figure 5. (a) 2-thermistor circuit from [1] (b) modified version.

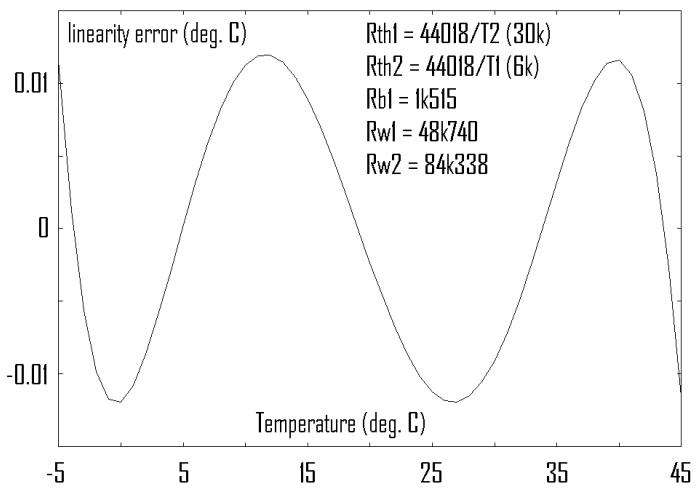

Figure 6. Calculated optimal error function for fig. 5(b).

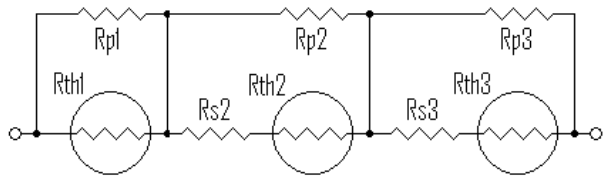

Figure 7. Experimental thermistor circuit.

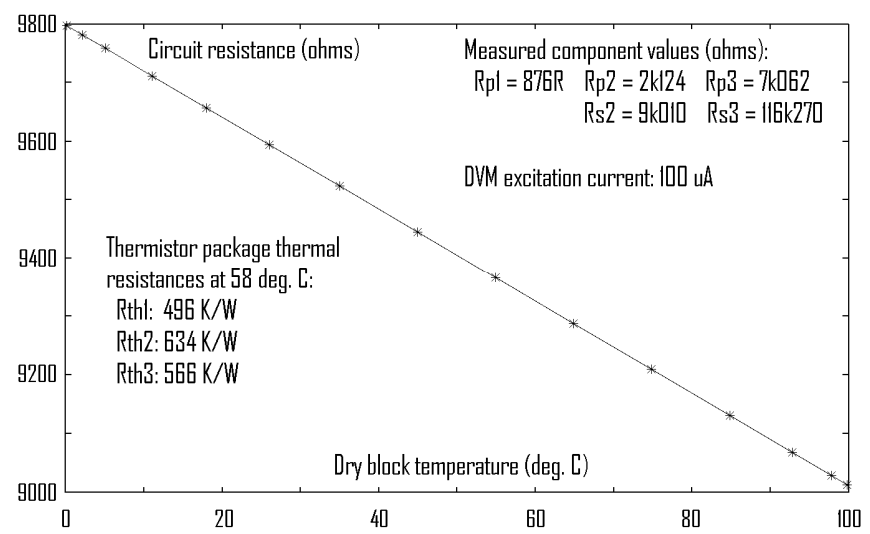

Figure 8. Measured resistance of experimental circuit in fig. 7.

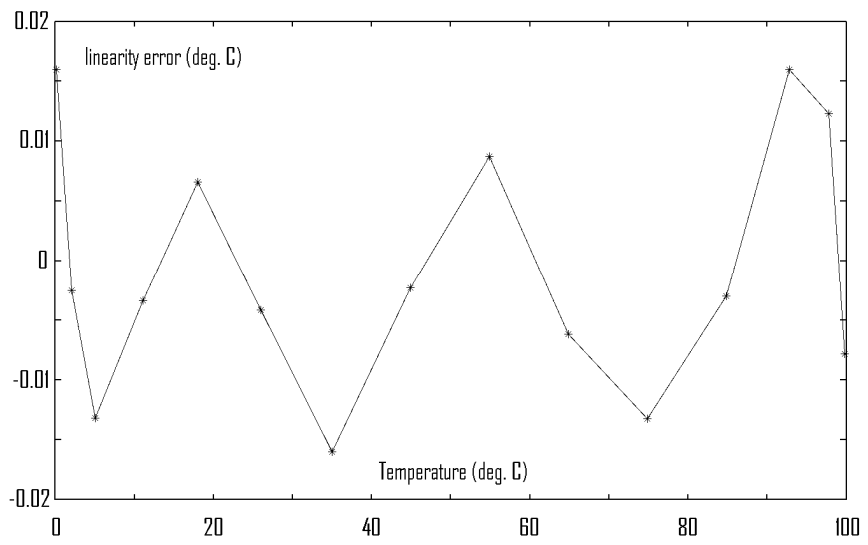

Figure 9. Measured linearity error in experimental circuit of fig. 7.

\section{REFERENCES}

[1] H. W. Trolander and R. W. Harruff, "Extended range thermistor temperature sensing," US Patent 3,316,765, May 1967.

[2] L. N. Nye, "Linearized thermistor networks," US Patent 3.652,921, March 1972.

[3] J. Helle, "Temperature compensation circuit for crystal oscillator," US Patent 4,020,426, April 1977

[4] S. Fry, "Temperature compensating circuit for a crystal oscillator," US Patent 6,362,699, March 2002.

[5] B. Gilbert, "The multi-tanh principle: a tutorial overview," IEEE J. Solid-State Circuits, vol. 33, no. 1, pp 2-17, 1998.

[6] J. Oehm, D. Pham-Stäbner, "Linear controlled temperature independent varactor circuitry," Proc. $28^{\text {th }}$ European Solid-State Circuits Conf., 2002, pp 143-146, ESSCIRC 2002.

[7] YSI Inc., YSI Temperature industrial products catalog, June 2000.

[8] P. P. Petrushev and V. A. Popov, Rational approximation of real functions, Encyclopedia of mathematics and its applications, vol. 28, G.-C. Rota, ed., Cambridge, 1987.

[9] T. J. Rivlin, An introduction to the approximation of functions, Dover, 1981.

[10] BetaTHERM Corp., BetaLinear device, November 2003. Available online as http://www. betatherm.com/datasheets/pdfs/PC344.pdf

[11] C. Renneberg, "A circuit and method for fitting the output of a sensor to a predetermined linear relationship," PCT application PCT/AU2006/ 000897, December 2006. Available on-line from http://www.wipo.int

[12] Z. Moroń, "Can analog circuits still increase sensors' smartness?," Sensors and Actuators A: Physical, vol. 68, no. 1-3, pp 474-479, 1998.

[13] D. W. Feldbaumer, J. A. Babcock, V. M. Mercier, C. K. Y. Chun, "Pulse current trimming of polysilicon resistors," IEEE Trans. Electron Devices, vol. 42, no. 4, pp 689-696, 1995.

[14] J. A. Babcock et al., "Precision electrical trimming of very low TCR poly-SiGe resistors," IEEE Electron Device Letters, vol. 21, no. 6, pp 283-285, 2000.

[15] C. B. Moler, Numerical computing with MATLAB, SIAM, 2004.

[16] M. Sapoff et al., "The exactness of fit of resistance-temperature data of thermistors with third-degree polynomials," Temperature, Its Measurement and Control in Science and Industry, vol. 5, J. F. Schooley, ed., AIP, 1982.

[17] J. A. Nelder and R. Mead, "A simplex method for function minimization,” Comput. J., vol. 7, pp 308-313, 1965.

[18] B. Boehm, "Convergence of best rational Tchebycheff approximations," Trans. Am. Math. Soc., vol. 115, pp. 388-399, March 1965. Available on-line from http://www.jstor.org 